\title{
Bacterial Cell-Cell Communication in the Host via RRNPP Peptide-Binding Regulators
}

\author{
David Perez-Pascual*t, Véronique Monnet and Rozenn Gardan \\ Micalis Institute, INRA, AgroParisTech, Université Paris-Saclay, Jouy-en-Josas, France
}

Human microbiomes are composed of complex and dense bacterial consortia. In these environments, bacteria are able to react quickly to change by coordinating their gene expression at the population level via small signaling molecules. In Gram-positive bacteria, cell-cell communication is mostly mediated by peptides that are released into the extracellular environment. Cell-cell communication based on these peptides is especially widespread in the group Firmicutes, in which they regulate a wide array of biological processes, including functions related to host-microbe interactions. Among the different agents of communication, the RRNPP family of cytoplasmic transcriptional regulators, together with their cognate re-internalized signaling peptides, represents a group of emerging importance. RRNPP members that have been studied so far are found mainly in species of bacilli, streptococci, and enterococci. These bacteria are characterized as both human commensal and pathogenic, and share different niches in the human body with other microorganisms. The goal of this mini-review is to present the current state of research on the biological relevance of RRNPP mechanisms in the context of the host, highlighting their specific roles in commensalism or virulence.

Keywords: quorum sensing, firmicutes, virulence, commensalism, cell-cell communication

\section{INTRODUCTION}

Specialty section:

This article was submitted to Microbial Physiology and Metabolism, a section of the journal Frontiers in Microbiology

Received: 29 January 2016 Accepted: 28 April 2016 Published: 20 May 2016

Citation:

Perez-PascualD, MonnetV and Gardan R (2016) Bacterial Cell-Cell Communication in the Host via RRNPP Peptide-Binding

Throughout the human body, all surfaces with portals to the exterior are covered in microbes (Foxman and Martin, 2015), mostly bacteria, which adapt to the specific environmental conditions of each niche by fighting, competing, or co-habiting with other bacteria and host cells.

In these kinds of complex consortia, bacteria have developed sophisticated communication mechanisms wherein signaling molecules are exported into the extracellular environment, accumulated, and then detected by a sensor protein. This sensing leads bacteria to modulate the expression of target genes, thus enabling them to escape from immune defense or attack the host (in the case of virulence) or compete with other bacteria (in commensalism).

In Firmicutes, cell-cell communication, or quorum sensing, is mostly mediated by peptides. Most of these peptides are encoded by short open reading frames but a few of them are produced by the proteolytic degradation of signal peptides of lipoproteins. All of them are then released into the extracellular environment (Antunes and Ferreira, 2009; Atkinson and Williams, 2009). Some of these peptides can be detected in the extracellular medium by twocomponent systems, while others are actively imported back into bacteria by the oligopeptide transporter Opp, at which point they interact with sensors in the cytoplasm. This interaction modulates the activity of the sensors and thus the expression of target genes (Rocha-Estrada et al., 2010; Cook and Federle, 2014; Monnet and Gardan, 2015). These sensors belong to the RRNPP 
family of cytoplasmic regulatory receptors (name given from the different sensors described: Rgg, Rap, NprR, PlcR, and PrgX). They are characterized at the structural level by tetratricopeptide repeats, which are involved in the regulator/peptide interaction (Declerck et al., 2007; Parashar et al., 2015). Rgg, NprR, PlcR, and PrgX are transcriptional regulators sensu stricto and will be examined in this review (Table 1), whereas the Rap phosphatases found in bacilli will not. RAP proteins have a phosphatase activity and regardless of this catalytic activity, they can also inhibit the transcriptional activity of response regulators of two component systems. They have been mainly studied in Bacillus subtilis and are involved in the control of sporulation, competence, and production of degradative enzymes and antibiotics (for review see Pottathil and Lazazzera, 2003).

Different RRNPP mechanisms have been deciphered in detail (Figure 1) and described in several recent reviews (Cook et al., 2013; Slamti et al., 2014; Fontaine et al., 2015). However, these mechanisms have been validated mostly in vitro, and very few studies have explored how they function in complex environments. The RRNPP members that have been studied so far, are found in bacilli, streptococci, or enterococci. Some of the species of interest are human opportunistic pathogens and share different niches in the human body with other bacteria. This mini-review will focus on the data available concerning the functioning of these mechanisms in the context of the host. In addition, we will examine whether these systems are linked to virulence or commensalism.

\section{PrgX/TraA REPRESSORS AND VIRULENCE IN ENTEROCOCCI}

Conjugation is a horizontal gene transfer mechanism that controls the transfer of genes from donor cells to recipient cells after contact. Conjugation of sex pheromone plasmids in Enterococcus faecalis is controlled by a transcriptional repressor in the PrgX/TraA family that is able to bind either an activating or a repressing peptide. The conjugation of the pCF10 plasmid has been studied in detail. Very briefly, when bound to the activating peptide cCF10, PrgX adopts a dimeric form, which allows the expression of $\operatorname{prg} Q$ and the conjugation genes. In this way, conjugation can occur between pCF10 of the donor and the recipient cell. Instead, when bound to the inhibitory peptide iCF10, the PrgX repressor adopts a tetrameric

TABLE 1 | RRNPP family of transcriptional regulators with their associated peptides and their biological roles.

\begin{tabular}{|c|c|c|c|}
\hline $\begin{array}{l}\text { Name of } \\
\text { regulator }\end{array}$ & Peptide & Group & $\begin{array}{l}\text { Role of } \\
\text { mechanism }\end{array}$ \\
\hline Rgg & $\begin{array}{l}\text { SHP, } \\
\text { ComS (XIP) }\end{array}$ & Streptococci & Commensalism/Virulence \\
\hline NprR & NprX & Bacilli & Necrotrophism \\
\hline PlcR & PapR & Bacilli & Virulence \\
\hline PrgX, TraA & $\begin{array}{l}\text { cCF10 } \\
\text { iCF10 }\end{array}$ & Enterococci & Virulence \\
\hline TprA & PhrA & Streptococci & Commensalism \\
\hline
\end{tabular}

conformation; this represses the transcription of $\operatorname{prgQ}$, which encodes iCF10, as well as that of downstream conjugation genes. Therefore, the conjugation cannot occur (Figure 1) (Clewell et al., 2014). This mechanism and additional regulatory pathways at the transcriptional and post-transcriptional levels have been thoroughly described in multiple recent reviews (Dunny, 2007, 2013; Dunny and Johnson, 2011).

E. faecalis is a commensal bacterium that colonizes the intestinal tract of humans as well as the vagina and oral cavity, but it is also an opportunistic pathogen which causes significant nosocomial infections. Over 20 plasmids belonging to the sex pheromone family have been found to be more prevalent in clinical enterococcal strains (Coque and Murray, 1995). In vivo conjugation has been reported in human plasma (Hirt et al., 2002) as well as in different animal models such as the gastrointestinal tract of hamsters, rabbit plasma, and the endocarditis or subdermal chamber in rabbits (Huycke et al., 1992; Hirt et al., 2002).

Part of the regulon of the PrgX system is the aggregation substance (AS), a surface protein that mediates donor/recipient attachment and which is encoded by the $\operatorname{prgB}$ gene. Interestingly, synthesis of AS is induced, through the PrgX mechanism, in donor cells in contact with human plasma, in the absence of recipient cells (Hirt et al., 2002). This observation led to the proposal of a model in which a plasma component, probably an albumin/lipid complex, inactivates the inhibitory peptide iCF10, thus allowing the expression of the conjugation genes, including $\operatorname{prgB}$ (Chandler et al., 2005). Beyond its role in conjugation, AS has been shown to be a virulence factor in different rabbit models of endocarditis; in some of these models it produces cardiac vegetations and higher mortality (Chow et al., 1993; Schlievert et al., 1998; Hirt et al., 2002). AS also accelerates early biofilm development in an ex vivo porcine heart valve model (ChuangSmith et al., 2010). It promotes adhesion to cultured renal tubular cells from pork (Kreft et al., 1992) and adhesion to and invasion of different types of cultured human enterocytes (Olmsted et al., 1994; Sartingen et al., 2000; Wells et al., 2000; Waters et al., 2003, 2004). Finally, AS also protects E. faecalis against attack by human neutrophils despite phagocytosis and neutrophil activation (Rakita et al., 2000) and by human macrophages (Sussmuth et al., 2000).

\section{PIcR/PapR AND VIRULENCE IN THE Bacillus cereus GROUP}

The B. cereus group comprises multiple species (Bottone, 2010), including the insect pathogen Bacillus thuringiensis, the etiological agent of anthrax Bacillus anthracis, (Kolsto et al., 2009), and B. cereus sensu stricto, an opportunistic pathogen causing food-borne gastroenteritis and systemic infections (Stenfors Arnesen et al., 2008). In B. cereus and B. thuringiensis, $\mathrm{PlcR}$ is a pleiotropic transcriptional activator that induces the expression of $80 \%$ of the genes coding for extracellular factors, including some virulence factors such as degradation enzymes and cytotoxic and cell surface proteins (Agaisse et al., 1999; Gohar et al., 2002, 2008). Interaction of the peptide PapR 


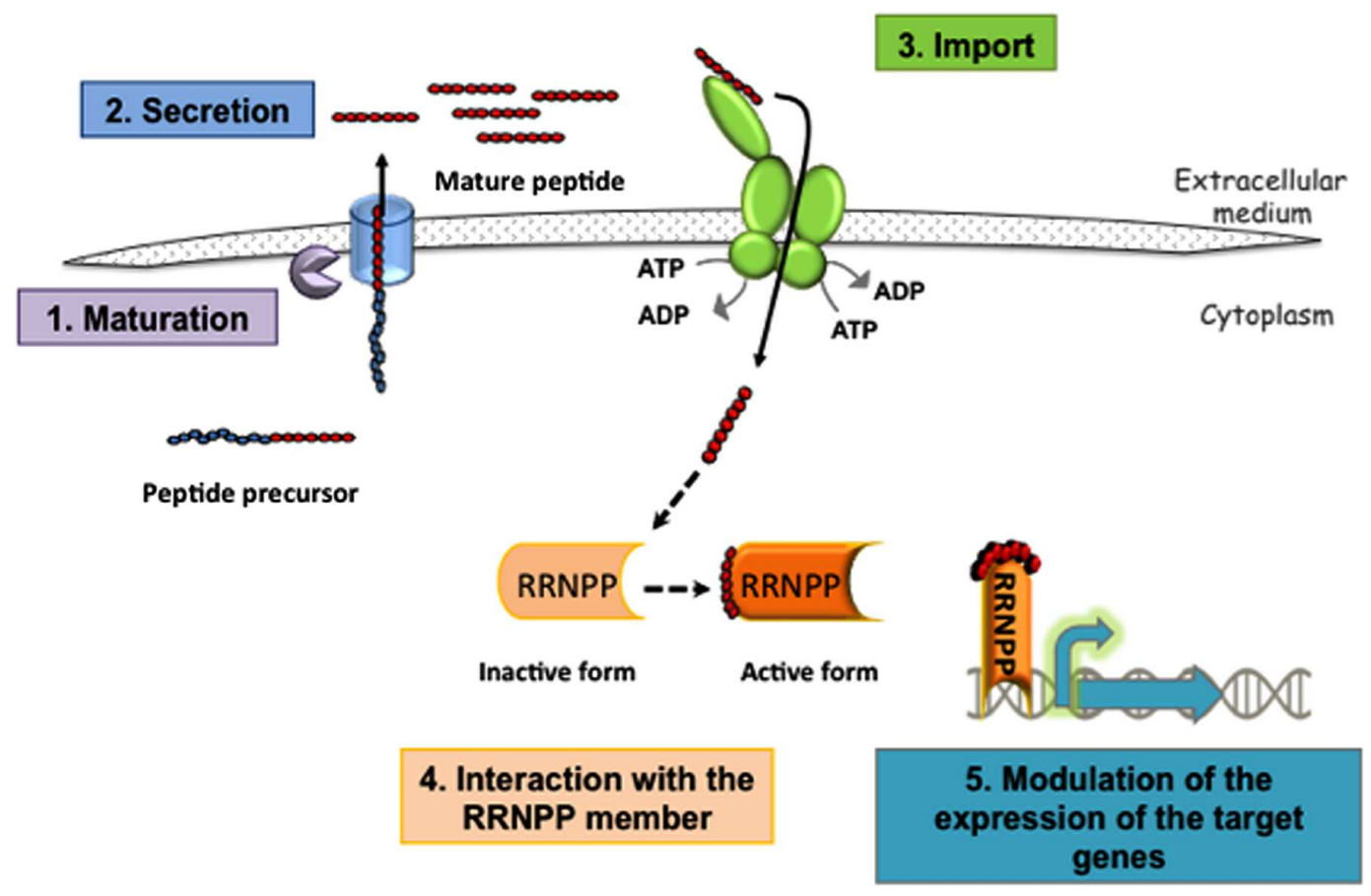

FIGURE 1 | Schematic representation of the RRNPP mechanism involving transcriptional regulators. (1) Maturation of a peptide precursor (or protein in E. faecalis) and (2) secretion of the mature peptide releases a signaling peptide outside the bacterium. These two processes involve various exporters and proteases, depending on the bacteria, and some have yet to be identified. The order in which these steps occur is also largely unknown. (3) Next, the signaling peptide is imported into the cell by Opp, a transporter from the ATP-binding cassette (ABC) family. (4) Finally, the signaling peptides interact with their specific sensors (Rgg, NprR, PlcR, or PrgX), and (5) modulate the expression of their target genes. For NprR, PlcR, or the Rgg which function as activators binding to their cognate peptide allows them to positively control the expression of their target genes. For TprA or the Rgg3 that function as repressor binding to their cognate peptide alleviates the repressor effect. The repressing function of $\mathrm{PrgX}$ is either strengthened by the binding of an inhibitory peptide or weakened by the binding of an activating peptide.

with PlcR allows the complex to bind to DNA and enables transcription of the target genes (Figure 1) (Grenha et al., 2013). In $B$. thuringiensis, disruption of the $p a p R$ gene inactivated the expression of the PlcR regulon, resulting in decreased hemolytic activity and a significant reduction in virulence in an insect infection model (Slamti and Lereclus, 2002).

Other studies have also identified a role for PlcR in the regulation of pathogenesis in $B$. cereus and B. thuringiensis. In both of these bacteria, PlcR inactivation provokes a significant reduction in virulence in mice (Salamitou et al., 2000) and rabbits (Callegan et al., 2003). Similarly, in ex vivo cell culture assays, cytotoxicity of B. thuringiensis strain Bt407 in Hela or Caco2 human epithelial cells was PlcR-dependent. In addition, this toxic effect was cell-contact-independent, which supports the hypothesis that at least one of the secreted factors regulated by $\mathrm{PlcR}$ is responsible for this cytotoxicity (Ramarao and Lereclus, 2006). Three important enterotoxins in the B. cereus group the hemolysin $\mathrm{BL}(\mathrm{Hbl})$, the cytotoxin $\mathrm{K}(\mathrm{CytK})$, and the nonhemolytic enterotoxin (Nhe) - are under control of the PlcRPapR system (Agaisse et al., 1999). Hbl and Nhe are pore-forming toxins, which induce cell lysis in different eukaryotic cell models (Jessberger et al., 2014) and CytK is cytotoxic toward the Caco2 intestinal cell line (Hardy et al., 2001; Fagerlund et al., 2004; Jessberger et al., 2014). More generally, it is hypothesized that these enterotoxins are responsible for the abdominal cramps and diarrhea that are symptoms of infection (Ramarao and Sanchis, 2013). However, plcR mutant strains are not completely avirulent (Tran et al., 2011), suggesting that other virulence factors non-regulated by $\mathrm{PlcR}$ play a role during $B$. cereus infections (Guillemet et al., 2010).

\section{NprR/NprX AND NECROTROPHISM IN THE B. cereus GROUP}

The NprR/NprX system appears to be functional in three of the species in this group: B. anthracis, B. cereus, and B. thuringiensis (Rice et al., 2015). The sensor NprR interacts with the signaling peptide NprX, and the complex then activates the expression of NprA metalloprotease (Perchat et al., 2011), as well as other genes that encode degradative enzymes like lipases or peptidases (Figure 1) (Dubois et al., 2012). Interestingly, the PlcR-PapR complex stimulates the transcription of the $n p r R-n \operatorname{pr} X$ operon (Dubois et al., 2013).

In B. thuringiensis, it has been proposed that this system participates in the necrotrophism process in an insect larval model (Dubois et al., 2012). However, little is known about its activity in the mammalian environment, with one exception: in $B$. anthracis, the $n p r R$ gene was highly expressed during the outgrowth of spores within murine macrophages (Bergman et al., 
2007), which suggests that this system plays a role in B. anthracis pathogenesis.

\section{Rgg/SHP IN COMMENSALISM AND VIRULENCE IN STREPTOCOCCI}

The Rgg transcriptional regulators are widespread among many species of the phylum Firmicutes. However, Rgg-associated peptides have only been found in Streptococcaceae thus far. Two subfamilies of Rgg are distinguished: (i) Rgg associated with the SHP (small hydrophobic peptides) family of peptides, which regulates genes of diverse functions, and (ii) the Rgg called ComR, which, in association with the ComS (XIP) family of peptides, controls the triggering of competence in multiple species (Ibrahim et al., 2007b; Fleuchot et al., 2011).

Peptide-associated Rgg mechanisms were first deciphered in Streptococcus thermophilus (Ibrahim et al., 2007a; Fontaine et al., 2010, 2013; Fleuchot et al., 2011) and later in three opportunistic pathogenic streptococci (Figure 1). The first of these was the human commensal associated with dental caries, Streptococcus mutans, which contains a ComS/ComR system (Mashburn-Warren et al., 2010). To our knowledge, no data are available in vivo for this bacterium. The second was the humanrestricted pathogen Streptococcus pyogenes (or GAS, from Group A Streptococcus), which has two interrelated SHP/Rgg systems, SHP2/Rgg2 and SHP3/Rgg3, in addition to a ComS/ComR system (Chang et al., 2011). More recently, the opportunistic human and animal pathogen Streptococcus agalactiae (or GBS, from Group $\underline{B}$ Streptococcus; Cook et al., 2013; Perez-Pascual et al., 2015) was determined to have one active SHP/Rgg, called SHP/RovS.

GAS is commonly carried asymptomatically in the oropharynx or on the skin but can become invasive and cause severe life-threatening diseases. The $\operatorname{sh} p 2 / r g g 2$ and $\operatorname{sh} p 3 / r g g 3$ loci are present in all sequenced strains of GAS and the complex Rgg2/3 pathway of strain NZ131 has recently been extensively studied in chemically defined medium (Lasarre et al., 2013; Cook and Federle, 2014). In addition to the shp2/3 genes, Rgg2/3 control the transcription of downstream genes whose function remains unclear. However, this pathway modulates the levels of biofilm produced by GAS (Chang et al., 2011). Moreover, SHP/Rgg signaling is induced considerably in the presence of mannose, one of the prominent carbohydrates present in the glycoconjugates on airway epithelia. In addition, these inducing conditions confer upon GAS resistance to lysozyme, an antimicrobial host defense mechanism present in mucosal secretions and in macrophages (Chang et al., 2015). Additional experiments also showed that the peptidase PepO, whose gene is negatively regulated by CovR, can degrade SHP2 and SHP3 and, therefore, inhibit SHP/Rgg signaling. CovR is a two-component system regulator that inhibits the expression of many genes, including those encoding potential virulence factors. Despite the lack of direct in vivo experimental evidence for the biological relevance of these SHP/Rgg systems in GAS, these results led the authors to hypothesize that the Rgg2/3 pathway more likely plays a role in asymptomatic carriage in the host (Wilkening et al.,
2015). However, in contrast to this hypothesis, another study in strain SF370 demonstrated that Rgg2 and its associated regulon were implicated in infection development, as the inactivation of $r g g 2$ decreased the ability of GAS to adhere to epithelial cells and increased biofilm formation. Additionally, a $\Delta r g g 2$ mutant strain was unable to grow in human blood and showed avirulent behavior in a murine model (Zutkis et al., 2014).

GBS is a commensal of the human intestinal and vaginal tract in $15-30 \%$ of healthy adults, but remains one of the most important invasive pathogens in newborns and the elderly (Le Doare and Heath, 2013). Nearly all sequenced GBS strains present a unique copy of the shp/rovs locus. This cell-cell communication mechanism has been recently deciphered (Cook et al., 2013; Perez-Pascual et al., 2015), demonstrating that the SHP/RovS mechanism plays an important role in bacterial pathogenesis. Mice infected by isogenic shp or rovs deletion mutants showed a significant decrease in the bacterial burden in the liver and spleen compared to mice infected by the parental strain. Further exploration revealed that disruption of shp and rovs resulted in a significant decrease in the ability of GBS to adhere to and invade human HepG2 hepatic cells (Perez-Pascual et al., 2015). In addition, in chemically defined medium, at least three genes are under the control of this mechanism: shp and $g b s 1556$ (encoding a secreted protein), which are positively and directly controlled by SHP/RovS, and the $f b s A$ gene, which codes for a fibrinogen-binding protein involved in GBS pathogenesis (Schubert et al., 2004) and is repressed by SHP/RovS. In rich medium, RovS also seems to control a set of genes related to virulence, such as $\operatorname{sod} A$ and $c y l E$, which encode a superoxide dismutase and a protein required for the production of hemolysin, respectively (Samen et al., 2006).

Another peptide-associated Rgg transcriptional regulator has been studied in Streptococcus suis (Zheng et al., 2011). In this bacterium, inactivation of Rgg attenuated pathogenicity in a piglet infection model, probably due to growth and metabolism changes in the mutant. Surprisingly, a $\Delta r g g$ mutant showed hyper-adhesion to epithelial cells and increased hemolytic activity. DNA microarray analysis revealed that Rgg is a global regulator that affects genes with varied functions, such as the metabolism of non-glucose carbohydrates (e.g., lactose or maltose) or defense mechanisms. The authors suggest that Rgg is a global transcriptional regulator that plays a relevant role in S. suis survival during pathogen-host interaction (Zheng et al., 2011).

In streptococci, the $\operatorname{com} X$ gene encodes an alternative sigma factor that controls the transcription of the genes necessary for natural transformation, and transcription of $\operatorname{com} X$ is either controlled by a two-component system or a peptide-associated Rgg-like regulator, the ComS/ComR system. The involvement of ComS/ComR in the triggering of transformation has been experimentally validated in laboratory conditions for four different species: S. mutans, S. thermophilus, Streptococcus infantarius, and Streptococcus macedonicus (Fontaine et al., 2015). Interestingly, in GAS, in spite of the fact that no natural transformation has been observed in many laboratory conditions (Mashburn-Warren et al., 2012), transformants were recovered at low levels from cells grown 
in biofilm on epithelial cells or in vivo from mice colonized intranasally with biofilm bacteria (Marks et al., 2014).

\section{TprA/PhrA AND COMMENSALISM IN Streptococcus pneumoniae}

Recently, new families of peptide-associated regulators have been identified in S. pneumoniae, which is commensal in the human nasopharynx, but also the etiological agent of serious diseases such as pneumonia, bacteremia, or meningitis (Shak et al., 2013). These new families comprise a transcriptional regulator, $\mathrm{Tpr}$, and its allied cognate peptide Phr (Hoover et al., 2015). Blast analysis revealed that this system appears to be orthologous to the PlcR/PapR system in bacilli. One such system, TprA/PhrA, regulates the expression of a putative lantibiotic biosynthesis operon. TprA represses the expression of its own encoding gene, as well as that of the phrA gene and the lantibiotic genes. However, the PhrA peptide antagonizes TprA activity. A study of this regulation following the expression of the $p h r A$ gene revealed that, whereas glucose can inhibit $p h r A$ expression, galactose, the main sugar of the human nasopharynx, can induce it (Hoover et al., 2015). Tn-seq analysis (sequencing of insertion transposon sites by high throughput sequencing methods) highlighted that disruptions of tprA or some of the lantibiotic genes reduced the fitness of S. pneumoniae for nasopharynx colonization in a murine model (van Opijnen and Camilli, 2012). These results led Hoover et al. (2015) to hypothesize that expression of the lantibiotic genes through the TprA/PhrA system may be important during niche colonization, where $S$. pneumoniae competes with other bacteria for space and resources. This was supported by the observation that the TprA/PhrA system and its regulated lantibiotic gene cluster are not needed for invasive disease development in a murine model (van Opijnen and Camilli, 2012; Hoover et al., 2015).

\section{CONCLUSIONS AND PERSPECTIVES}

In Firmicutes, peptides are the best-known signaling molecules. The peptides that interact with RRNPP regulators are short ( $<10$ amino acids), without post-translational modifications, are secreted and then reimported into bacteria through an oligopeptide transporter. The RRNPP transcriptional regulators are activated by these peptides via tetratricopeptide domains involved in peptide-protein interactions. RRNPP regulators and reimported short peptides act together as a communication system, which clearly differs from a second group of communication systems. In this second group, which includes the well-documented Agr system from Staphylococcus aureus, peptides are post-translationally modified and activate two components systems at the outside bacterial surface.

The number of putative members of the RRNPP family has increased dramatically; for example, in streptococci, the $\mathrm{Tpr} / \mathrm{Phr}$ and $\mathrm{Rgg} / \mathrm{SHP}$ families are predicted to have 53 and 68 members, respectively, (Fleuchot et al., 2011; Hoover et al., 2015) and it is highly probable that others remain to be discovered. In order to better understand these regulators, much work remains, especially focusing on their role and in vivo function in opportunistic pathogens. Research conducted thus far indicates that RRNPP members regulate a variety of biological functions. For example, PlcR/PapR as well as $\operatorname{PrgX} / \operatorname{TraA}$ have a direct impact on virulence, through controlling the expression of target genes that encode virulence factors. The TprA/PhrA system has been implicated in the commensal behavior of S. pneumoniae and seems to be involved in the expression of a bacteriocin-encoding operon. The role of Rgg/SHP mechanisms is more complex. Whereas the RovS/SHP system in GBS seems to participate in disease development, the orthologous mechanism in GAS, Rgg2/SHP2, has been predominantly linked to commensal behavior. Moreover, this system is expressed at variable levels in different strains of GAS (Chang et al., 2015).

Existing conclusions regarding the regulatory function of RRNPP members in the host are derived from indirect results and require further validation. In particular, this field would benefit from in vivo imaging of the induction of these mechanisms during host colonization. Furthermore, gene expression studies at the single-cell level are rare; to our knowledge, only one such study exists, an investigation of PlcR and NprR of B. thuringiensis in an insect model (Verplaetse et al., 2015). These innovative approaches should greatly improve the identification of host-associated environmental conditions that induce these mechanisms.

Finally, when viewed in the context of emerging resistance to antibiotics, the RRNPP systems represent an attractive target for research into new mechanisms for bacterial control. In the future, it is possible that infections by some opportunistic pathogens could be prevented by decreasing the expression of virulence factors, without altering the composition of the global microbiota, via modulation of the activity of RRNPP regulators.

\section{AUTHOR CONTRIBUTIONS}

All authors listed, have made substantial, direct and intellectual contribution to the work, and approved it for publication.

\section{FUNDING}

This study was supported by the Marie Curie Intra-European Fellowship for Career Development (IEF) No. 327146 and the DIM-ASTREA grant from the Region Ile-de-France (Grant No. ast110095).

\section{ACKNOWLEDGMENT}

We are grateful to N. Rama Rao for help, advice, and critical reading of the manuscript. 


\section{REFERENCES}

Agaisse, H., Gominet, M., Okstad, O. A., Kolsto, A. B., and Lereclus, D. (1999). $\mathrm{PlcR}$ is a pleiotropic regulator of extracellular virulence factor gene expression in Bacillus thuringiensis. Mol. Microbiol. 32, 1043-1053. doi: 10.1046/j.13652958.1999.01419.x

Antunes, L. C., and Ferreira, R. B. (2009). Intercellular communication in bacteria. Crit. Rev. Microbiol. 35, 69-80. doi: 10.1080/10408410902 733946

Atkinson, S., and Williams, P. (2009). Quorum sensing and social networking in the microbial world. J. R. Soc. Interface 6, 959-978. doi: 10.1098/rsif. 2009.0203

Bergman, N. H., Anderson, E. C., Swenson, E. E., Janes, B. K., Fisher, N., Niemeyer, M. M., et al. (2007). Transcriptional profiling of Bacillus anthracis during infection of host macrophages. Infect. Immun. 75, 3434-3444. doi: 10.1128/IAI.01345-06

Bottone, E. J. (2010). Bacillus cereus, a volatile human pathogen. Clin. Microbiol. Rev. 23, 382-398. doi: 10.1128/CMR.00073-09

Callegan, M. C., Kane, S. T., Cochran, D. C., Gilmore, M. S., Gominet, M., and Lereclus, D. (2003). Relationship of plcR-regulated factors to Bacillus endophthalmitis virulence. Infect. Immun. 71, 3116-3124. doi: 10.1128/IAI.71.6.3116-3124.2003

Chandler, J. R., Flynn, A. R., Bryan, E. M., and Dunny, G. M. (2005). Specific control of endogenous CCF10 pheromone by a conserved domain of the pCF10encoded regulatory protein PrgY in Enterococcus faecalis. J. Bacteriol. 187, 4830-4843. doi: 10.1128/JB.187.14.4830-4843.2005

Chang, J. C., Jimenez, J. C., and Federle, M. J. (2015). Induction of a quorum sensing pathway by environmental signals enhances group A streptococcal resistance to lysozyme. Mol. Microbiol. 97, 1097-1113. doi: 10.1111/mmi. 13088

Chang, J. C., LaSarre, B., Jimenez, J. C., Aggarwal, C., and Federle, M. J. (2011). Two group A streptococcal peptide pheromones act through opposing Rgg regulators to control biofilm development. PLoS Pathog. 7:e1002190. doi: 10.1371/journal.ppat.1002190

Chow, J. W., Perri, M. B., Thal, L. A., and Zervos, M. J. (1993). Mobilization of the penicillinase gene in Enterococcus faecalis. Antimicrob. Agents Chemother. 37, 1187-1189. doi: 10.1128/AAC.37.5.1187

Chuang-Smith, O. N., Wells, C. L., Henry-Stanley, M. J., and Dunny, G. M. (2010). Acceleration of Enterococcus faecalis biofilm formation by aggregation substance expression in an ex vivo model of cardiac valve colonization. PLoS ONE 5:e15798. doi: 10.1371/journal.pone.0015798

Clewell, D. B., Weaver, K. E., Dunny, G. M., Coque, T. M., Francia, M. V., and Hayes, F. (2014). "Extrachromosomal and mobile elements in Enterococci: transmission, maintenance, and epidemiology," in Enterococci: From Commensals to Leading Causes of Drug Resistant Infection, eds M. S. Gilmore, D. B. Clewell, Y. Ike, and N. Shankar (Boston, MA: Massachusetts Eye and Ear Infirmary).

Cook, L. C., and Federle, M. J. (2014). Peptide pheromone signaling in Streptococcus and Enterococcus. FEMS Microbiol. Rev. 38, 473-492. doi: 10.1111/1574-6976.12046

Cook, L. C., LaSarre, B., and Federle, M. J. (2013). Interspecies communication among commensal and pathogenic streptococci. MBio 4:e00382-13. doi: 10.1128/mBio.00382-13

Coque, T. M., and Murray, B. E. (1995). Identification of Enterococcus faecalis strains by DNA hybridization and pulsed-field gel electrophoresis. J. Clin. Microbiol. 33, 3368-3369.

Declerck, N., Bouillaut, L., Chaix, D., Rugani, N., Slamti, L., Hoh, F., et al. (2007). Structure of PlcR: insights into virulence regulation and evolution of quorum sensing in Gram-positive bacteria. Proc. Natl. Acad. Sci. U.S.A. 104, 18490-18495. doi: 10.1073/pnas.0704501104

Dubois, T., Faegri, K., Perchat, S., Lemy, C., Buisson, C., Nielsen-LeRoux, C., et al. (2012). Necrotrophism is a quorum-sensing-regulated lifestyle in Bacillus thuringiensis. PLoS Pathog. 8:e1002629. doi: 10.1371/journal.ppat.1002629

Dubois, T., Perchat, S., Verplaetse, E., Gominet, M., Lemy, C., AumontNicaise, M., et al. (2013). Activity of the Bacillus thuringiensis NprR-NprX cell-cell communication system is co-ordinated to the physiological stage through a complex transcriptional regulation. Mol. Microbiol. 88, 48-63. doi: $10.1111 / \mathrm{mmi} .12168$
Dunny, G. M. (2007). The peptide pheromone-inducible conjugation system of Enterococcus faecalis plasmid pCF10: cell-cell signalling, gene transfer, complexity and evolution. Philos. Trans. R. Soc. Lond. B Biol. Sci. 362, 11851193. doi: 10.1098/rstb.2007.2043

Dunny, G. M. (2013). Enterococcal sex pheromones: signaling, social behavior, and evolution. Annu. Rev. Genet. 47, 457-482. doi: 10.1146/annurev-genet-111212133449

Dunny, G. M., and Johnson, C. M. (2011). Regulatory circuits controlling enterococcal conjugation: lessons for functional genomics. Curr. Opin. Microbiol. 14, 174-180. doi: 10.1016/j.mib.2011.01.008

Fagerlund, A., Ween, O., Lund, T., Hardy, S. P., and Granum, P. E. (2004). Genetic and functional analysis of the cytK family of genes in Bacillus cereus. Microbiology 150, 2689-2697. doi: 10.1099/mic.0.26975-0

Fleuchot, B., Gitton, C., Guillot, A., Vidic, J., Nicolas, P., Besset, C., et al. (2011). Rgg proteins associated with internalized small hydrophobic peptides: a new quorum-sensing mechanism in streptococci. Mol. Microbiol. 80, 1102-1119. doi: 10.1111/j.1365-2958.2011.07633.x

Fontaine, L., Boutry, C., de Frahan, M. H., Delplace, B., Fremaux, C., Horvath, P., et al. (2010). A novel pheromone quorum-sensing system controls the development of natural competence in Streptococcus thermophilus and Streptococcus salivarius. J. Bacteriol. 192, 1444-1454. doi: 10.1128/JB. 01251-09

Fontaine, L., Goffin, P., Dubout, H., Delplace, B., Baulard, A., Lecat-Guillet, N., et al. (2013). Mechanism of competence activation by the ComRS signalling system in streptococci. Mol. Microbiol. 87, 1113-1132. doi: 10.1111/mmi. 12157

Fontaine, L., Wahl, A., Flechard, M., Mignolet, J., and Hols, P. (2015). Regulation of competence for natural transformation in streptococci. Infect. Genet. Evol. 33, 343-360. doi: 10.1016/j.meegid.2014.09.010

Foxman, B., and Martin, E. T. (2015). Use of the microbiome in the practice of epidemiology: a primer on -omic technologies. Am. J. Epidemiol. 182, 1-8. doi: 10.1093/aje/kwv102

Gohar, M., Faegri, K., Perchat, S., Ravnum, S., Okstad, O. A., Gominet, M., et al. (2008). The PlcR virulence regulon of Bacillus cereus. PLoS ONE 3:e2793. doi: 10.1371/journal.pone.0002793

Gohar, M., Okstad, O. A., Gilois, N., Sanchis, V., Kolsto, A. B., and Lereclus, D. (2002). Two-dimensional electrophoresis analysis of the extracellular proteome of Bacillus cereus reveals the importance of the $\mathrm{PlcR}$ regulon. Proteomics 2, 784-791. doi: 10.1002/1615-9861(200206)2:6<784::AID-PROT784>3.0. CO;2-R

Grenha, R., Slamti, L., Nicaise, M., Refes, Y., Lereclus, D., and Nessler, S. (2013). Structural basis for the activation mechanism of the PlcR virulence regulator by the quorum-sensing signal peptide PapR. Proc. Natl. Acad. Sci. U.S.A. 110, 1047-1052. doi: 10.1073/pnas.1213770110

Guillemet, E., Cadot, C., Tran, S. L., Guinebretiere, M. H., Lereclus, D., and Ramarao, N. (2010). The InhA metalloproteases of Bacillus cereus contribute concomitantly to virulence. J. Bacteriol. 192, 286-294. doi: 10.1128/JB. 00264-09

Hardy, S. P., Lund, T., and Granum, P. E. (2001). CytK toxin of Bacillus cereus forms pores in planar lipid bilayers and is cytotoxic to intestinal epithelia. FEMS Microbiol. Lett. 197, 47-51. doi: 10.1111/j.1574-6968.2001.tb 10581.x

Hirt, H., Schlievert, P. M., and Dunny, G. M. (2002). In vivo induction of virulence and antibiotic resistance transfer in Enterococcus faecalis mediated by the sex pheromone-sensing system of pCF10. Infect. Immun. 70, 716-723. doi: 10.1128/IAI.70.2.716-723.2002

Hoover, S. E., Perez, A. J., Tsui, H. C., Sinha, D., Smiley, D. L., DiMarchi, R. D., et al. (2015). A new quorum-sensing system (TprA/PhrA) for Streptococcus pneumoniae D39 that regulates a lantibiotic biosynthesis gene cluster. Mol. Microbiol. 97, 229-243. doi: 10.1111/mmi.13029

Huycke, M. M., Gilmore, M. S., Jett, B. D., and Booth, J. L. (1992). Transfer of pheromone-inducible plasmids between Enterococcus faecalis in the Syrian hamster gastrointestinal tract. J. Infect. Dis. 166, 1188-1191. doi: 10.1093/infdis/166.5.1188

Ibrahim, M., Guillot, A., Wessner, F., Algaron, F., Besset, C., Courtin, P., et al. (2007a). Control of the transcription of a short gene encoding a cyclic peptide in Streptococcus thermophilus: a new quorum-sensing system? J. Bacteriol. 189, 8844-8854. doi: 10.1128/JB.01057-07 
Ibrahim, M., Nicolas, P., Bessieres, P., Bolotin, A., Monnet, V., and Gardan, R. (2007b). A genome-wide survey of short coding sequences in streptococci. Microbiology 153, 3631-3644. doi: 10.1099/mic.0.2007/0 06205-0

Jessberger, N., Dietrich, R., Bock, S., Didier, A., and Martlbauer, E. (2014). Bacillus cereus enterotoxins act as major virulence factors and exhibit distinct cytotoxicity to different human cell lines. Toxicon 77, 49-57. doi: 10.1016/j.toxicon.2013.10.028

Kolsto, A. B., Tourasse, N. J., and Okstad, O. A. (2009). What sets Bacillus anthracis apart from other Bacillus species? Annu. Rev. Microbiol. 63, 451-476. doi: 10.1146/annurev.micro.091208.073255

Kreft, B., Marre, R., Schramm, U., and Wirth, R. (1992). Aggregation substance of Enterococcus faecalis mediates adhesion to cultured renal tubular cells. Infect. Immun. 60, 25-30.

Lasarre, B., Aggarwal, C., and Federle, M. J. (2013). Antagonistic Rgg regulators mediate quorum sensing via competitive DNA binding in Streptococcus pyogenes. MBio 3:e00333-12. doi: 10.1128/mBio.00333-12

Le Doare, K., and Heath, P. T. (2013). An overview of global GBS epidemiology. Vaccine 31(Suppl. 4), D7-D12. doi: 10.1016/j.vaccine.2013.01.009

Marks, L. R., Mashburn-Warren, L., Federle, M. J., and Hakansson, A. P. (2014). Streptococcus pyogenes biofilm growth in vitro and in vivo and its role in colonization, virulence, and genetic exchange. J. Infect. Dis. 210, 25-34. doi: 10.1093/infdis/jiu058

Mashburn-Warren, L., Morrison, D. A., and Federle, M. J. (2010). A novel double-tryptophan peptide pheromone controls competence in Streptococcus spp. via an Rgg regulator. Mol. Microbiol. 78, 589-606. doi: 10.1111/j.13652958.2010.07361.x

Mashburn-Warren, L., Morrison, D. A., and Federle, M. J. (2012). The cryptic competence pathway in Streptococcus pyogenes is controlled by a peptide pheromone. J. Bacteriol. 194, 4589-4600. doi: 10.1128/JB.00830-12

Monnet, V., and Gardan, R. (2015). Quorum-sensing regulators in Grampositive bacteria: 'cherchez le peptide.' Mol. Microbiol. 97, 181-184. doi 10.1111/mmi.13060

Olmsted, S. B., Dunny, G. M., Erlandsen, S. L., and Wells, C. L. (1994). A plasmidencoded surface protein on Enterococcus faecalis augments its internalization by cultured intestinal epithelial cells. J. Infect. Dis. 170, 1549-1556. doi: 10.1093/infdis/170.6.1549

Parashar, V., Aggarwal, C., Federle, M. J., and Neiditch, M. B. (2015). Rgg protein structure-function and inhibition by cyclic peptide compounds. Proc. Natl. Acad. Sci. U.S.A. 112, 5177-5182. doi: 10.1073/pnas.1500357112

Perchat, S., Dubois, T., Zouhir, S., Gominet, M., Poncet, S., Lemy, C., et al. (2011). A cell-cell communication system regulates protease production during sporulation in bacteria of the Bacillus cereus group. Mol. Microbiol. 82, 619-633. doi: 10.1111/j.1365-2958.2011.07839.x

Perez-Pascual, D., Gaudu, P., Fleuchot, B., Besset, C., Rosinski-Chupin, I., Guillot, A., et al. (2015). RovS and its associated signaling peptide form a cell-tocell communication system required for Streptococcus agalactiae pathogenesis. MBio 6:e02306-14. doi: 10.1128/mBio.02306-14

Pottathil, M., and Lazazzera, B. A. (2003). The extracellular Phr peptide-Rap phosphatase signaling circuit of Bacillus subtilis. Front. Biosci. 8, d32-d45. doi: $10.2741 / 913$

Rakita, R. M., Quan, V. C., Jacques-Palaz, K., Singh, K. V., Arduino, R. C., Mee, M., et al. (2000). Specific antibody promotes opsonization and PMN-mediated killing of phagocytosis-resistant Enterococcus faecium. FEMS Immunol. Med. Microbiol. 28, 291-299. doi: 10.1111/j.1574-695X.2000.tb01489.x

Ramarao, N., and Lereclus, D. (2006). Adhesion and cytotoxicity of Bacillus cereus and Bacillus thuringiensis to epithelial cells are FlhA and PlcR dependent, respectively. Microbes Infect. 8, 1483-1491. doi: 10.1016/j.micinf.2006. 01.005

Ramarao, N., and Sanchis, V. (2013). The pore-forming haemolysins of Bacillus cereus: a review. Toxins (Basel) 5, 1119-1139. doi: 10.3390/toxins 5061119

Rice, A. J., Woo, J. K., Khan, A., Szypulinski, M. Z., Johnson, M. E., Lee, H., et al. (2015). Over-expression, purification, and confirmation of Bacillus anthracis transcriptional regulator NprR. Protein Expr. Purif. doi: 10.1016/j.pep.2015.08.030 [Epub ahead of print]

Rocha-Estrada, J., Aceves-Diez, A. E., Guarneros, G., and de la Torre, M. (2010). The RNPP family of quorum-sensing proteins in Gram-positive bacteria. Appl. Microbiol. Biotechnol. 87, 913-923. doi: 10.1007/s00253-0102651-y

Salamitou, S., Ramisse, F., Brehelin, M., Bourguet, D., Gilois, N., Gominet, M., et al. (2000). The plcR regulon is involved in the opportunistic properties of Bacillus thuringiensis and Bacillus cereus in mice and insects. Microbiology 146, 2825-2832. doi: 10.1099/00221287-146-11-2825

Samen, U. M., Eikmanns, B. J., and Reinscheid, D. J. (2006). The transcriptional regulator RovS controls the attachment of Streptococcus agalactiae to human epithelial cells and the expression of virulence genes. Infect. Immun. 74, 56255635. doi: 10.1128/IAI.00667-06

Sartingen, S., Rozdzinski, E., Muscholl-Silberhorn, A., and Marre, R. (2000). Aggregation substance increases adherence and internalization, but not translocation, of Enterococcus faecalis through different intestinal epithelial cells in vitro. Infect. Immun. 68, 6044-6047. doi: 10.1128/IAI.68.10.60446047.2000

Schlievert, P. M., Gahr, P. J., Assimacopoulos, A. P., Dinges, M. M., Stoehr, J. A., Harmala, J. W., et al. (1998). Aggregation and binding substances enhance pathogenicity in rabbit models of Enterococcus faecalis endocarditis. Infect. Immun. 66, 218-223.

Schubert, A., Zakikhany, K., Pietrocola, G., Meinke, A., Speziale, P., Eikmanns, B. J., et al. (2004). The fibrinogen receptor FbsA promotes adherence of Streptococcus agalactiae to human epithelial cells. Infect. Immun. 72, 6197-6205. doi: 10.1128/IAI.72.11.6197-6205.2004

Shak, J. R., Ludewick, H. P., Howery, K. E., Sakai, F., Yi, H., Harvey, R. M., et al. (2013). Novel role for the Streptococcus pneumoniae toxin pneumolysin in the assembly of biofilms. MBio 4:e00655-13. doi: $10.1128 / \mathrm{mBio}$. 00655-13

Slamti, L., and Lereclus, D. (2002). A cell-cell signaling peptide activates the $\mathrm{PlcR}$ virulence regulon in bacteria of the Bacillus cereus group. EMBO J. 21, 4550-4559. doi: 10.1093/emboj/cdf450

Slamti, L., Perchat, S., Huillet, E., and Lereclus, D. (2014). Quorum sensing in Bacillus thuringiensis is required for completion of a full infectious cycle in the insect. Toxins (Basel) 6, 2239-2255. doi: 10.3390/toxins60 82239

Stenfors Arnesen, L. P., Fagerlund, A., and Granum, P. E. (2008). From soil to gut: Bacillus cereus and its food poisoning toxins. FEMS Microbiol. Rev. 32, 579-606. doi: 10.1111/j.1574-6976.2008.00112.x

Sussmuth, S. D., Muscholl-Silberhorn, A., Wirth, R., Susa, M., Marre, R., and Rozdzinski, E. (2000). Aggregation substance promotes adherence, phagocytosis, and intracellular survival of Enterococcus faecalis within human macrophages and suppresses respiratory burst. Infect. Immun. 68, 4900-4906. doi: 10.1128/IAI.68.9.4900-4906.2000

Tran, S. L., Guillemet, E., Ngo-Camus, M., Clybouw, C., Puhar, A., Moris, A. et al. (2011). Haemolysin II is a Bacillus cereus virulence factor that induces apoptosis of macrophages. Cell Microbiol. 13, 92-108. doi: 10.1111/j.14625822.2010.01522.x

van Opijnen, T., and Camilli, A. (2012). A fine scale phenotype-genotype virulence map of a bacterial pathogen. Genome Res. 22, 2541-2551. doi: $10.1101 /$ gr. 137430.112

Verplaetse, E., Slamti, L., Gohar, M., and Lereclus, D. (2015). Cell differentiation in a Bacillus thuringiensis population during planktonic growth, biofilm formation, and host infection. MBio 6:e00138-15. doi: $10.1128 / \mathrm{mBio}$ 00138-15

Waters, C. M., Antiporta, M. H., Murray, B. E., and Dunny, G. M. (2003). Role of the Enterococcus faecalis GelE protease in determination of cellular chain length, supernatant pheromone levels, and degradation of fibrin and misfolded surface proteins. J. Bacteriol. 185, 3613-3623. doi: 10.1128/JB.185.12.36133623.2003

Waters, C. M., Hirt, H., McCormick, J. K., Schlievert, P. M., Wells, C. L., and Dunny, G. M. (2004). An amino-terminal domain of Enterococcus faecalis aggregation substance is required for aggregation, bacterial internalization by epithelial cells and binding to lipoteichoic acid. Mol. Microbiol. 52, 1159-1171. doi: 10.1111/j.1365-2958.2004.04045.x

Wells, C. L., Moore, E. A., Hoag, J. A., Hirt, H., Dunny, G. M., and Erlandsen, S. L. (2000). Inducible expression of Enterococcus faecalis aggregation substance surface protein facilitates bacterial internalization by cultured enterocytes. Infect. Immun. 68, 7190-7194. doi: 10.1128/IAI.68.12.71907194.2000 
Wilkening, R. V., Chang, J. C., and Federle, M. J. (2015). PepO, a CovRScontrolled endopeptidase, disrupts Streptococcus pyogenes quorum sensing. Mol. Microbiol. 99, 71-87. doi: 10.1111/mmi.13216

Zheng, F., Ji, H., Cao, M., Wang, C., Feng, Y., Li, M., et al. (2011). Contribution of the Rgg transcription regulator to metabolism and virulence of Streptococcus suis serotype 2. Infect. Immun. 79, 1319-1328. doi: 10.1128/IAI.00193-10

Zutkis, A. A., Anbalagan, S., Chaussee, M. S., and Dmitriev, A. V. (2014). Inactivation of the Rgg2 transcriptional regulator ablates the virulence of Streptococcus pyogenes. PLoS ONE 9:e114784. doi: 10.1371/journal.pone.0114784
Conflict of Interest Statement: The authors declare that the research was conducted in the absence of any commercial or financial relationships that could be construed as a potential conflict of interest.

Copyright (c) 2016 Perez-Pascual, Monnet and Gardan. This is an open-access article distributed under the terms of the Creative Commons Attribution License (CC BY). The use, distribution or reproduction in other forums is permitted, provided the original author(s) or licensor are credited and that the original publication in this journal is cited, in accordance with accepted academic practice. No use, distribution or reproduction is permitted which does not comply with these terms. 